This item was submitted to Loughborough's Research Repository by the author.

Items in Figshare are protected by copyright, with all rights reserved, unless otherwise indicated.

\title{
Electrically efficient production of a diffuse nonthermal atmospheric plasma
}

PLEASE CITE THE PUBLISHED VERSION

PUBLISHER

(C) IEEE

VERSION

VoR (Version of Record)

LICENCE

CC BY-NC-ND 4.0

REPOSITORY RECORD

Deng, Xu-Tao, and Michael G. Kong. 2019. "Electrically Efficient Production of a Diffuse Nonthermal Atmospheric Plasma”. figshare. https://hdl.handle.net/2134/5222. 
This item was submitted to Loughborough's Institutional Repository (https://dspace.lboro.ac.uk/) by the author and is made available under the following Creative Commons Licence conditions.

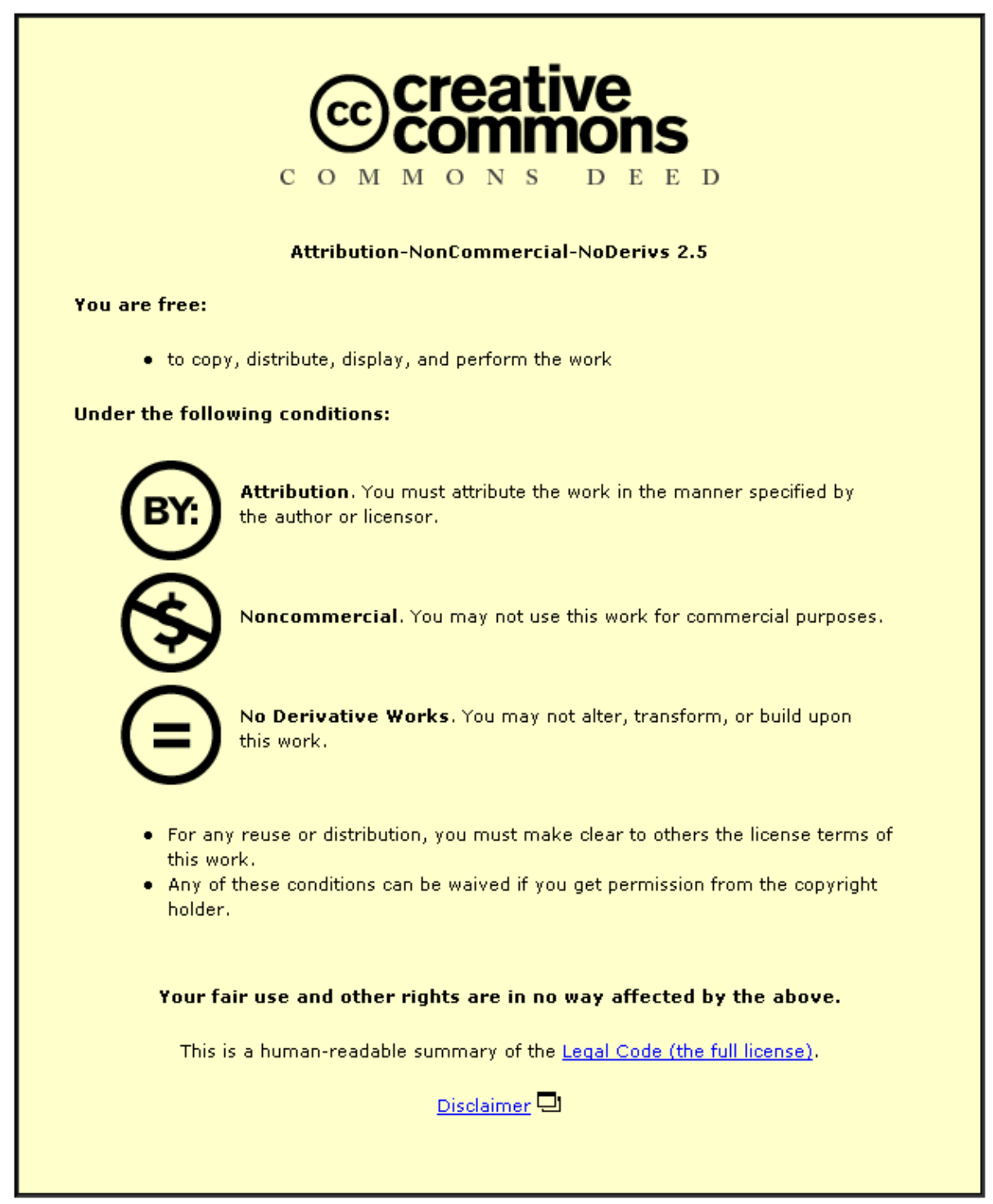

For the full text of this licence, please go to: http://creativecommons.org/licenses/by-nc-nd/2.5/ 


\title{
Electrically Efficient Production of a Diffuse Nonthermal Atmospheric Plasma
}

\author{
Michael G. Kong and Xu Tao Deng
}

Invited Paper

\begin{abstract}
Diffuse nonthermal gas discharges generated at atmospheric pressure have found increasing applications in many key materials processing areas such as etching, deposition, and structural modification of polymeric surfaces. To facilitate tailored and improved applications of these novel gas plasmas, we consider their pulsed generation based on one-dimensional numerical simulation of helium-nitrogen discharges. We consider four waveforms of the plasma-generating voltage, namely: 1) sinusoidal; 2) peaklevelled sinusoidal; 3) peak-levelled and tail-trimmed sinusoidal; and 4) pulsed with a Gaussian-shaped tail, all at the same repetition frequency of $10 \mathrm{kHz}$. For each case, voltage and current characteristics are calculated and then used to assess whether the generated plasma is diffuse and nonthermal. Densities of electrons, ions, and metastables are calculated, together with the dissipated electric power in the plasma bulk. It is found that plasma pulsing can significantly reduce the electric power needed to sustain diffuse nonthermal atmospheric plasmas. Specifically by choosing appropriate pulse shape, the plasma-sustaining power can be reduced by more than $50 \%$ without reducing densities of electrons, ions, and metastables. On the other hand, electron density can be enhanced by $68 \%$ with the same input electric power if the pulsewidth is suitably narrowed.
\end{abstract}

Index Terms-Atmospheric pressure nonthermal plasmas, gas discharge, plasma modeling, pulse plasmas.

\section{INTRODUCTION}

$\mathbf{N}$ ONTHERMAL gas discharges generated at atmospheric pressure have been the subject of active research largely because of their widespread use for ozone production [1], pollution control [2], and surface modification [3]. Typically, such atmospheric plasma consists of many nanosecond-scale microdischarges of streamer-like filaments [4] and there is a large body of work in literature on their fundamental properties and their applications [1]-[7]. Recently, it has been suggested that nonthermal atmospheric gas discharges can also be diffuse and luminous with the duration of their discharge current pulses in excess of submilliseconds [8]-[12]. Compared to their counterparts in the streamer-dominated mode, these diffuse nonthermal atmospheric plasmas have greater spatial uniformity, better temporal stability, and much lower gas temperature typically in the $75{ }^{\circ} \mathrm{C}-150{ }^{\circ} \mathrm{C}$ range [13]. These properties

Manuscript received May 28, 2002; revised October 15, 2002.

The authors are with the Department of Electronic and Electrical Engineering, Loughborough University, Leicestershire LE11 3TU, U.K.

Digital Object Identifier 10.1109/TPS.2003.808884 make them particularly attractive for a number of key materials processing applications such as etching, deposition, and structural modification of polymeric surfaces [8]-[13]. Diffuse nonthermal atmospheric plasmas have been generated with dielectrically insulated electrodes at audio frequencies [10], with uninsulated electrodes at radio frequencies [13], and at microwave frequencies [9]. Apart from their desirable spatial and temporal characteristics, diffuse nonthermal atmospheric plasmas have different electrical and chemical properties from that of their streamer-dominated counterparts. While many of their fundamental properties remain to be fully understood [8], [13], the focus of the field has been largely experimental improvement of existing applications and empirical exploration of new applications [8]-[13].

For controlled and optimized performance of material processing, it is always desirable to be able to adjust and tailor plasma properties. In the case of diffuse nonthermal atmospheric plasmas, this has been pursued mainly through plasma rig designs and gas composition, and to a lesser extent through an appropriate choice of the excitation frequency [14]. In this paper, we consider pulsing plasma excitation voltage as a way to control and improve properties of diffuse nonthermal atmospheric plasmas. For streamer-dominated atmospheric gas discharges, pulsed-plasma generation has been known to facilitate better energy efficiency and greater control of the glow-to-arc transition [1], [2]. If similar improvements can be achieved for diffuse nonthermal atmospheric plasmas, it will be useful for applications where plasma power consumption is an important issue, for example aircraft cloaking and industry-scale surface treatment.

As a first step to unravel benefits of plasma pulsing in different plasma generation configurations [9], [10], [13], we will consider nonthermal atmospheric gas discharges generated in a helium-nitrogen mixture and between two dielectrically insulated parallel-plate electrodes. The emphasis of this paper is on electrical properties of diffuse nonthermal atmospheric plasmas under pulsed excitation. Specifically, this paper attempts to answer: 1) whether pulsed plasma generation can lead to saving in electrical power needed to sustain the generated plasma and 2) if so how pulse shape and width may be adjusted to enhance energy efficiency. Our study is based on a one-dimensional (1-D) computer code [15] developed using a hydrodynamic model similar to those employed in most 
TABLE I

REACTIONS CONSIDERED FOR AN He- $\mathrm{N}_{2}$ DischaRgE AND THEIR RATE COEFFICIENTS

\begin{tabular}{|c|c|c|c|}
\hline No & Reaction & Reaction rate & Reference \\
\hline \multicolumn{4}{|c|}{ Direct ionization } \\
\hline 1 & $\mathrm{He}+\mathrm{e} \rightarrow \mathrm{He}^{+}+\mathrm{e}+\mathrm{e}$ & $\alpha_{1}$ & {$[10][18][19]$} \\
\hline 2 & $\mathrm{~N}_{2}+\mathrm{e} \rightarrow \mathrm{N}_{2}{ }^{+}+\mathrm{e}+\mathrm{e}$ & $\alpha_{2}$ & {$[10][20]$} \\
\hline \multicolumn{4}{|c|}{ Excitation } \\
\hline 3 & $\mathrm{He}+\mathrm{e} \rightarrow \mathrm{He}\left(2^{3} \mathrm{~S}\right)+\mathrm{e}$ & $\beta_{1}$ & {$[21]$} \\
\hline 4 & $\mathrm{He}+\mathrm{e} \rightarrow \mathrm{He}\left(2^{1} \mathrm{~S}\right)+\mathrm{e}$ & $\beta_{2}$ & [21] \\
\hline 5 & $\mathrm{He}\left(2^{3} \mathrm{~S}\right)+2 \mathrm{He}\left(1^{1} \mathrm{~S}\right) \rightarrow \mathrm{He}_{2}^{*}+\mathrm{He}\left(1^{1} \mathrm{~S}\right)$ & $1.9 \times 10^{-34} \mathrm{~cm}^{6} / \mathrm{s}$ & {$[22]$} \\
\hline \multicolumn{4}{|c|}{ De-excitation } \\
\hline 6 & $\mathrm{He}\left(2^{3} \mathrm{~S}\right)+\mathrm{e} \rightarrow \mathrm{He}\left(1^{1} \mathrm{~S}\right)+\mathrm{e}$ & $4.2 \times 10^{-9} \mathrm{~cm}^{3} / \mathrm{s}$ & [23] \\
\hline \multicolumn{4}{|c|}{ Penning ionization } \\
\hline 7 & $\mathrm{He}\left(2^{3} \mathrm{~S}\right)+\mathrm{N}_{2} \rightarrow \mathrm{He}\left(1^{1} \mathrm{~S}\right)+\mathrm{N}_{2}{ }^{+}+\mathrm{e}$ & $8 \times 10^{-11} \mathrm{~cm}^{3} / \mathrm{s}$ & {$[21][24]$} \\
\hline \multicolumn{4}{|c|}{ Stepwise ionization } \\
\hline 8 & $\mathrm{He}\left(2^{3} \mathrm{~S}\right)+\mathrm{He}\left(2^{3} \mathrm{~S}\right) \rightarrow \mathrm{He}\left(1^{1} \mathrm{~S}\right)+\mathrm{He}^{+}+\mathrm{e}$ & $2.9 \times 10^{-9} \mathrm{~cm}^{3} / \mathrm{s}$ & {$[25]$} \\
\hline \multicolumn{4}{|c|}{ Charge transfer } \\
\hline 9 & $\mathrm{He}^{+}+2 \mathrm{He}\left(1^{1} \mathrm{~S}\right) \rightarrow \mathrm{He}_{2}^{+}+\mathrm{He}\left(1^{1} \mathrm{~S}\right)$ & $6.3 \times 10^{-32} \mathrm{~cm}^{6} / \mathrm{s}$ & {$[16][21]$} \\
\hline \multicolumn{4}{|c|}{ Recombination } \\
\hline 10 & $\mathrm{He}^{+}+\mathrm{e} \rightarrow \mathrm{He}\left(1^{1} \mathrm{~S}\right)$ & $2 \times 10^{-12} \mathrm{~cm}^{3} / \mathrm{s}$ & {$[26]$} \\
\hline 11 & $\mathrm{He}_{2}{ }^{+}+\mathrm{e} \rightarrow \mathrm{He}_{2}{ }^{*}$ & $5 \times 10^{-16} \mathrm{~cm}^{3} / \mathrm{s}$ & {$[27]$} \\
\hline 12 & $\mathrm{He}^{+}+\mathrm{e}+\mathrm{He} \rightarrow \mathrm{He}\left(1^{1} \mathrm{~S}\right)+\mathrm{He}\left(2^{3} \mathrm{~S}\right)$ & $1 \times 10^{-27} \mathrm{~cm}^{6} / \mathrm{s}$ & [26] \\
\hline 13 & $\mathrm{He}_{2}^{+}+2 \mathrm{e} \rightarrow \mathrm{He}\left(1^{1} \mathrm{~S}\right)+\mathrm{e}$ & $7.1 \times 10^{-20} \mathrm{~cm}^{6} / \mathrm{s}$ & {$[16][21]$} \\
\hline 14 & $\mathrm{He}_{2}{ }^{+}+\mathrm{e} \rightarrow 2 \mathrm{He}\left(2^{3} \mathrm{~S}\right)+\mathrm{He}$ & $5 \times 10^{-9} \mathrm{~cm}^{3} / \mathrm{s}$ & [22] \\
\hline 15 & $\mathrm{He}_{2}{ }^{+}+\mathrm{e}+\mathrm{He} \rightarrow \mathrm{He}_{2}{ }^{*}+\mathrm{He}$ & $5 \times 10^{-27} \mathrm{~cm}^{6} / \mathrm{s}$ & {$[27]$} \\
\hline 16 & $\mathrm{~N}_{2}{ }^{+}+\mathrm{e} \rightarrow \mathrm{N}+\mathrm{N}$ & $4.8 \times 10^{-8} \mathrm{~cm}^{3} / \mathrm{s}$ & {$[24]$} \\
\hline 17 & $\mathrm{~N}_{2}{ }^{+}+2 \mathrm{e} \rightarrow \mathrm{N}_{2}+\mathrm{e}$ & $1.4 \times 10^{-26} \mathrm{~cm}^{6} / \mathrm{s}$ & {$[28]$} \\
\hline
\end{tabular}

numerical studies for diffuse nonthermal atmospheric plasmas [10], [16]. In Section II, we will review key features of our physical model of diffuse nonthermal atmospheric plasmas as well as its numerical implementation. Voltage-current $(V-I)$ characteristics of nonthermal atmospheric plasma under sinusoidal excitation will be discussed through numerical examples. In Section III, two different waveforms of pulsed excitation voltage will be considered and the $V-I$ characteristics of the generated atmospheric plasmas simulated to compare their power efficiency with that under sinusoidal excitation. Furthermore densities of electrons, ions, and metastables will be computed and compared with that obtained for the sinusoidal case. Also studied are effects of pulse duration, again through $V-I$ characteristic, plasma power density, and densities of electrons, ions, and metastables. Finally, in Section IV, findings from our numerical study will be summarized.

\section{Physical Model of Nonthermal ATMOSPHERIC PLASMAS}

Diffuse nonthermal atmospheric plasmas considered here are induced and sustained between two parallel-plate electrodes, each coated with a dielectric layer and connected externally to a kilovolt sinusoidal voltage source at audio frequencies. The background gas is atmospheric helium mixed with a small fraction of nitrogen (up to $1 \%$ ) at room temperature of $293 \mathrm{~K}$, and the dynamics of the generated nonthermal atmospheric plasma is described by the Boltzmann equations. The nitrogen fraction reflects the amount of impurities often found in industrial grade pure helium gases, and much larger nitrogen content, for example greater than 5\%, was found numerically to lower the glow-arc transition criterion as a result of enhanced Penning ionization. For diffuse nonthermal atmospheric plasmas, it has been established that the hydrodynamic assumptions are applicable [10], [16]. This reduces the Boltzmann equations to the continuity and momentum transfer equations for electrons and ions, thus facilitating a macroscopic description of plasma dynamics without its microscopic details [10], [15]. On the other hand, the dynamics of electrons and ions are determined by the electric field in the space between the two parallel electrodes, which has two components, one induced by the externally applied excitation voltage and the other by space charges. The electric field can be calculated by solving Poisson's equation. In the 1-D limit, the Boltzmann equations and Poisson's equations are closely coupled together as follows:

$$
\begin{aligned}
\frac{\partial n_{ \pm}}{\partial t}= & S_{ \pm}(\vec{r}, t)-\frac{\partial\left[n_{ \pm}(\vec{r}, t) W_{ \pm}(\vec{r}, t)\right]}{\partial \vec{r}} \\
& +\frac{\partial^{2}\left[n_{ \pm}(\vec{r}, t) D_{ \pm}(\vec{r}, t)\right]}{\partial \vec{r}^{2}}
\end{aligned}
$$




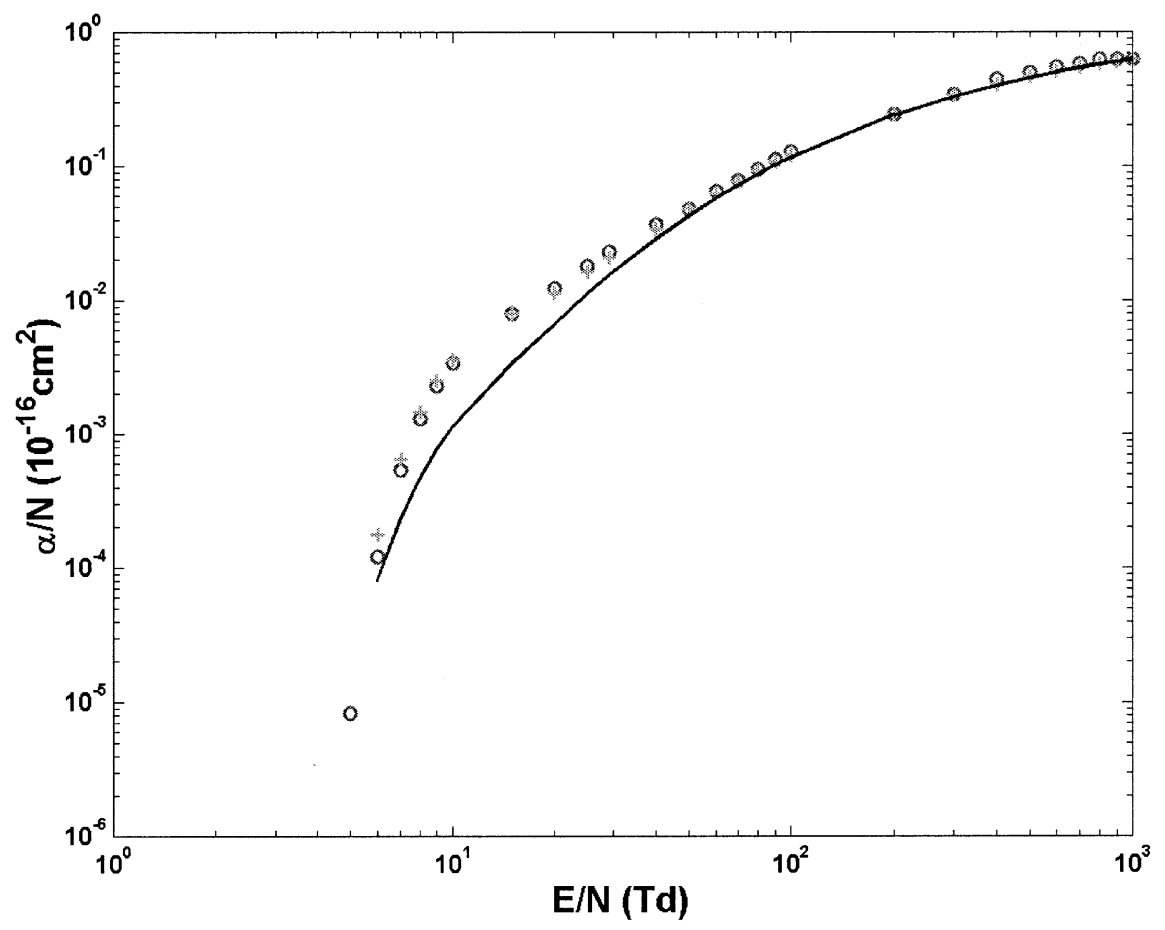

Fig. 1. Reduced ionization coefficient of argon as computed with a Boltzmann solver (circles) [10] and with the source term technique ( + ) [20] together with reduced ionization coefficient of nitrogen using the source term technique (solid line) [20].

$$
\begin{aligned}
\frac{\partial n_{i}}{\partial t}= & S_{i}(\vec{r}, t)-\frac{\partial\left[n_{i}(\vec{r}, t) W_{i}(\vec{r}, t)\right]}{\partial \vec{r}} \\
& +\frac{\partial^{2}\left[n_{i}(\vec{r}, t) D_{i}(\vec{r}, t)\right]}{\partial \vec{r}^{2}} \\
\frac{\partial E(\vec{r}, t)}{\partial r}= & \frac{|e|}{\varepsilon_{0}}\left[n_{+}(\vec{r}, t)-n_{-}(r, t)\right]
\end{aligned}
$$

where $n_{+}$and $n_{-}$are the ion and electron densities, respectively, and $n_{i}$ is the density of the $i$ th neutral species considered. $S_{ \pm}$and $S_{i}$ are the source terms for charged particles and neutral species, respectively. $D$ and $W$ are diffusion coefficient and drift velocity with subscripts,-+ , and $i$ denoting, respectively, electrons, ions, and $i$ th neutral species considered in the physical model. With the hydrodynamic assumptions, reaction rate coefficients, ionization coefficients, drift velocity, and diffusion coefficients can be approximated as a function of the electric field in the ionized gas between the two electrodes. Thus, our physical model is similar to those used in [10], [16]. The values of these coefficients are obtained from relevant experiments and several rate compilation studies which will be discussed in greater details below. These allow (1a) and (1b) to be solved to yield densities of electrons, ionic species, and metastables, which then allows through (1c) calculation of electric field. Boundary conditions between the ionized gas and the dielectrically coated electrodes need to be carefully treated. To this end, a circuit equation is included to relate the electric field in the gas to the source voltage (the output voltage of the power supply) via a source resistor, $R_{s}$, and two serial capacitors each representing one dielectric coating layer. Numerical algorithm used to solve the above equations is essentially based on the Pantankar scheme, and our discretization employs the upwind scheme [17].

Our model considers reactions participated by eight different species, namely,

1) two ground-state neutral species- $\mathrm{He}\left(1^{1} \mathrm{~S}\right)$ and $\mathrm{N}_{2}\left(4^{0} \mathrm{~S}\right)$;

2) two helium metastables- $\mathrm{He}\left(2^{3} \mathrm{~S}\right)$ and $\mathrm{He}\left(2^{1} \mathrm{~S}\right)$;

3) electrons;

4) ground-state atomic helium ions, $\mathrm{He}^{+}$;

5) ground-state molecular helium ions, $\mathrm{He}_{2}+\left(2^{3} \mathrm{~S} \Sigma_{u}^{+}\right)$;

6) ground state molecular nitrogen ions, $\mathrm{N}_{2}^{+}$.

In total, our model considers 17 reactions, as detailed in Table I, together with their reaction rates and reference sources. Products of these 17 reactions include three additional species-excited molecular helium $\left(\mathrm{He}^{*}\right)$, atomic nitrogen $(\mathrm{N})$, and molecular nitrogen ions $\left(\mathrm{N}_{2}^{+}\right)$. Any subsequent reaction of these three additional species with the original eight species is not considered in the model. It is worth noting that our model is capable of evaluating production of molecular nitrogen ions that are not considered in reported numerical studies of diffuse nonthermal atmospheric discharges [10], [16].

Ionization coefficient in helium can be evaluated using Ward's formula [18] or experimental data compiled in [19]. Both agree well with those used in [10], particularly at large reduced electric field $(>50$ Td). Our model uses Ward's formula. On the other hand, it is known from spectroscopic measurements that discharge dynamics in atmospheric helium is very strongly affected by the presence of impuries, particularly nitrogen [10]. For diffuse nonthermal atmospheric helium discharges, the presence of nitrogen impuries has been either ignored [16] or implemented with its ionization coefficient 


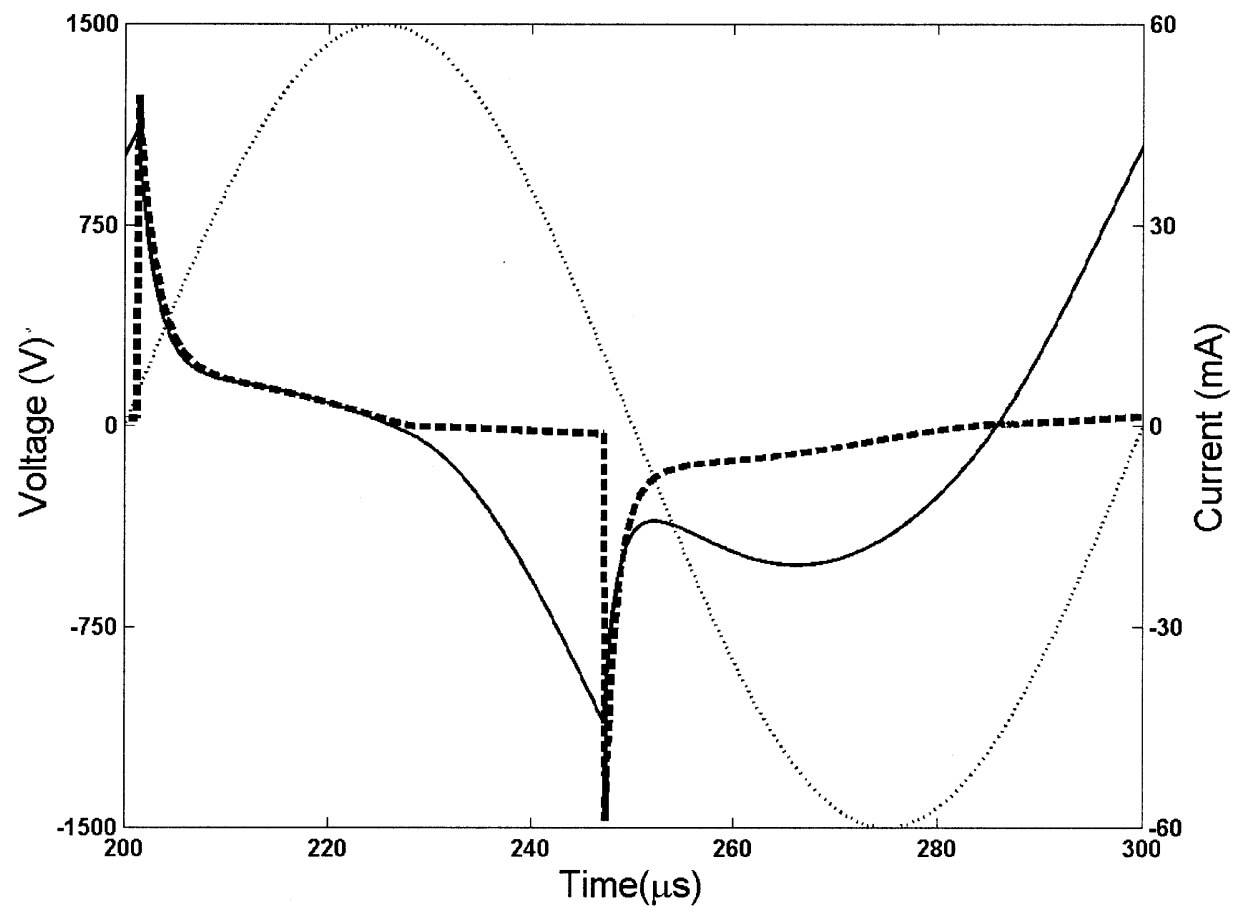

Fig. 2. Typical $V-I$ characteristics of a helium-nitrogen discharge under sinusoidal excitation with the gas voltage in solid curve, the discharge current in thick dashed curve, and the applied voltage in dot curve.

approximated by that of argon [10]. Since argon has a greater ionization coefficient than nitrogen [18], [19], the approximation of using argon ionization coefficient overestimates the ionization of nitrogen impurities. To this end, we note that it is possible to formulate analytically ionization source terms for discharges in argon, helium, and nitrogen [20]. Thus, under the same conditions

$$
\frac{\alpha_{A}}{\alpha_{B}}=\frac{\text { ionization source term for species A }}{\text { ionization source term for species B }}
$$

where $\alpha$ is Townsend's first ionization coefficient with its subscript $A$ and $B$ indicating, respectively, species $A$ and $B$. If species $A$ is argon and species $B$ is helium, the formula can be used to predict the ionization coefficient of argon from that of helium as calculated from Ward's formula [18]. As shown in Fig. 1, the argon ionization coefficient calculated with this technique leads to an excellent agreement with that computed using a Boltzmann solver [10]. Therefore, (2) offers a simple yet reliable way to estimate the ionization coefficient of one gas from the known ionization coefficient of a reference gas. Similarly, if we assume species $A$ is nitrogen and $B$ is helium, the ionization coefficient of nitrogen is obtained and this is shown in Fig. 1 as a function of the reduced electric field. Ionization coefficient thus calculated is used in our model for nitrogen (reaction \#2 in Table I). For excitation coefficients, we use that employed in [21]. Similarly, our model employs the same data used in [21] for diffusion coefficients and drift velocity.

For numerical examples presented here, the two electrodes have a common radius of $2 \mathrm{~cm}$ and are separated with a gap fixed at $0.5 \mathrm{~cm}$. The total capacitance of the dielectric coatings on the two parallel-plate electrodes is $70 \mathrm{pF}$, and the source resistor in the external circuit is $50 \Omega$. As the reference case, we consider a sinusoidal source voltage at $10 \mathrm{kHz}$ with a peak voltage of $1.5 \mathrm{kV}$. Before the power supply is switched onto the plasma rig, the two electrodes are charged at $950 \mathrm{~V}$ and so the initial gas voltage is $950 \mathrm{~V}$. The background gas is $99.5 \%$ helium with $0.5 \%$ nitrogen unless otherwise stated, and its breakdown voltage is assumed to be $1.1 \mathrm{kV}$, similar to the choice in [10]. The secondary electron emission coefficient is dependent on the surface condition of electrodes and, as such, it is not possible to choose a reliable coefficient that is applicable to most cases. Numerically different secondary emission coefficients in the 0.01-0.2 range affect the peak value of the discharge current. For numerical studies considered here, we choose 0.2 for atomic helium ions, 0.1 for molecular helium ions, and 0.01 for nitrogen ions [29]. Our numerical model assumes that diffuse nonthermal atmospheric plasma is established if its voltage and current are continuous and repetitive over at least 10 cycles of the applied voltage signal. Noting that discharge currents of both thermal plasmas and streamer-dominated nonthermal atmospheric plasmas tend to exhibit microscopic current pulses in tens of nanoseconds or less, pulse duration of the discharge current is an additional indicator for diffuse atmospheric plasmas. Fig. 2 shows a plot of the applied voltage, gas voltage, and discharge current as a function of time. Though not shown in Fig. 2, numerical results confirm that the discharge current is, in fact, identical through many tens of cycles of the applied voltage and so the discharge plasma is stable and repetitive temporally over a long period of time. The $V-I$ characteristics are very similar to that obtained in a comparable experiment and its numerical simulation [10], with a typical pattern of one discharge every half cycle of the applied voltage. Both gas voltage and discharge current exhibit 
very similar waveforms as those observed in [10], [16]. The peak discharge current in Fig. 2 is between 52-58 mA, lower than $90 \mathrm{~mA}$ calculated in [10]. As shown in Fig. 1, this may be partly due to the use of the larger ionization coefficient of argon in [10] to approximate the nitrogen ionization coefficient. It is interesting to note that the peak current density of $7.2 \mathrm{~mA} / \mathrm{cm}^{2}$ $\left(=90 \mathrm{~mA} / \pi 2^{2}\right)$ is the highest reported for diffuse atmospheric helium discharges generated between dielectrically coated electrodes and at kilohertz frequencies. In a series of very similar experiments performed at the University of Tennessee, the peak current density is found at most $1 \mathrm{~mA} / \mathrm{cm}^{2}$ [30]. A similar peak current density of about $0.4 \mathrm{~mA} / \mathrm{cm}^{2}\left(=11.25 \mathrm{~mA} / \pi 3^{2}\right)$ was measured with $3-\mathrm{kHz}$ plasma excitation by the Okazaki Group, Sophia University, Japan [31], whereas another Japanese group measured $1.8 \mathrm{~mA} / \mathrm{cm}^{2}$ with a $100-\mathrm{kHz}$ power supply. More recently, a helium discharge experiment performed at the University of Minnesota recorded a peak current density measured at $0.5 \mathrm{~mA} / \mathrm{cm}^{2}$ with $15-\mathrm{kHz}$ driving voltage [32] Our computed current density of $4.4 \mathrm{~mA} / \mathrm{cm}^{2}\left(=55 \mathrm{~mA} / \pi 2^{2}\right)$ falls between these experimental measurements, indicating a good current prediction capability of our model.

Electric power consumption in the plasma is found to be $298 \mathrm{~mW} / \mathrm{cm}^{3}$ from our model. This is almost identical to $300 \mathrm{~mW} / \mathrm{cm}^{3}$ measured in [10] and similar to $277 \mathrm{~mW} / \mathrm{cm}^{3}$ measured in [33]. We have also calculated electron density and ion density across the space between the two electrodes. Fig. 3 shows the time and spatial variation of electron density, similar to that computed in [10]. The rapid rise of electron density near $250 \mu \mathrm{s}$ is a result of the gas ionization (or discharge) in that half cycle (250-300 $\mu \mathrm{s})$, and the relatively uncharged electron density peak during the remaining period of the half cycle suggests a phase of inactive electron production. The instantaneous maximum electron density predicted with our model is $0.95 \times 10^{10} \mathrm{~cm}^{-3}$, not dissimilar to the instantaneous maximum of $2 \times 10^{10} \mathrm{~cm}^{-3}$ in [16], but much lower than the instantaneous maximum of $30 \times 10^{10} \mathrm{~cm}^{-3}$ calculated in [10]. Again, the large electron density reported in [10] may be a result of their overestimated ionization coefficient for nitrogen (see Fig. 1). For diffuse atmospheric helium plasmas, there is very little data of direct measurement of electron density for comparison with our calculated electron density. However indirectly, the ion-trapping mechanism proposed by Roth may be used to estimate the average electron density and this can be captured by the following [30]:

$$
n_{e}=\frac{2 m v_{c} P_{\mathrm{av}}}{e^{2} E_{0}^{2}}
$$

where $n_{e}$ is the average electron density, $m$ is the electron mass, $e$ is the electron charge, $v_{c}$ is the electron collision frequency in helium, $P_{\mathrm{av}}$ is the average electric power density consumed in the plasma, and $E_{0}$ is the peak electric field in the plasma. Assuming $P_{\mathrm{av}}=300 \mathrm{~mW} / \mathrm{cm}^{3}, E_{0}=3 \mathrm{kV} / \mathrm{cm}$, and $v_{c}=$ $1.8 \times 10^{12} \mathrm{~Hz}$ [30], (3) yields $4.3 \times 10^{8} \mathrm{~cm}^{-3}$. Given that the peak electron density is approximately 10-50 times greater than the average electron density as indicated in Fig. 3, the above calculation suggests that the peak electron density is between

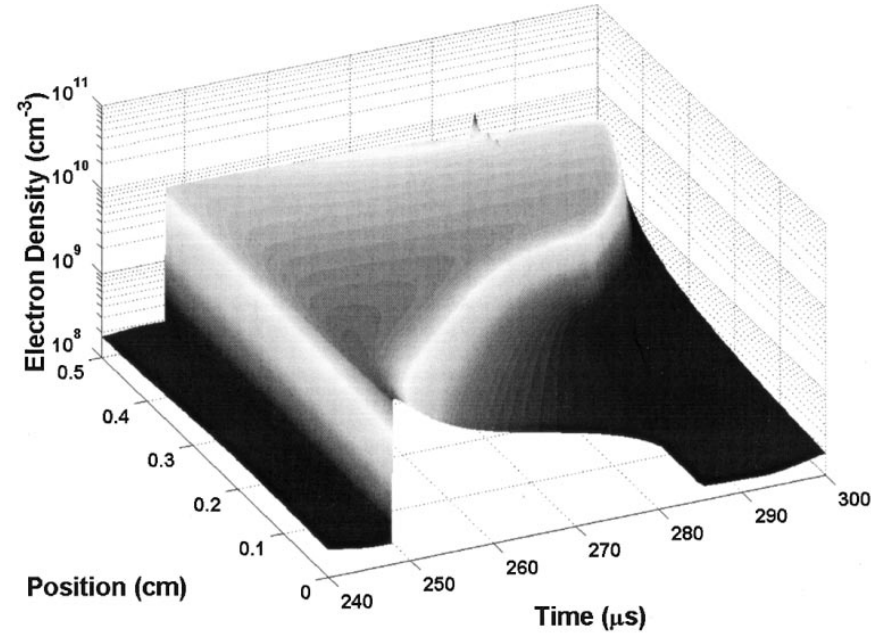

Fig. 3. Variation of electron density between the two electrodes over one-half cycle of the applied sinusoidal voltage. The instantaneous anode and cathode are at $x=0$ and $x=0.5 \mathrm{~cm}$, respectively, from $t=250$ to $t=300 \mu \mathrm{s}$.

$4 \times 10^{9} \mathrm{~cm}^{-3}$ and $2 \times 10^{10} \mathrm{~cm}^{-3}$. Thus, our calculated peak electron density appears reasonable. This is also confirmed by electron density estimate using the current density formula of $J=e n_{e} \mu E$ ( $\mu$ is the electron mobility), as employed for measuring electron density in microhollow cathode discharges [34]. In general, our simulated $V-I$ characteristics, the peak discharge current density, the plasma power density, and the electron density yield a favorable comparison with available experimental and numerical data of comparable diffuse atmospheric helium discharges. While there is much scope to include other features of diffuse atmospheric plasmas, for example multidimensional effects, our plasma model is capable of capturing the main plasma features accurately and as such it will be used to explore the benefits of plasma pulsing.

\section{EFfects OF Plasma Pulsing}

The potential benefit of energy saving is best understood from the $V-I$ characteristic in Fig. 2 . It is seen that the discharge current is of very low magnitude after its peak between 207-245 $\mu$ s in the half-cycle from 200 to $250 \mu$ s, when the applied voltage is very large. This suggests that between the instant of this discharge current pulse and the polarity reversal point of the applied voltage in the same half cycle, the applied voltage does not necessarily contribute to plasma generation (see Fig. 2), but may adversely heat up electrons. Through energy transfer from electrons to heavy particles, this undesirable electron heating would increase gas temperature. Therefore, by reducing the excitation voltage over this "inactive" period of electron production, the gas temperature may be lowered and, equally importantly, the input power can be reduced. We first consider a simple pulsed excitation voltage waveform, shown in Fig. 4(a), which is essentially derived from a sinusoidal wave with its peak (positive and negative) levelled to a flat top. The voltage waveform of Fig. 4(a) can be easily obtained electronically, and so it represents a realistic option. The magnitude of the original sinusoidal voltage $V_{s}$ is fixed at $1.5 \mathrm{kV}$, whereas that of the peak-leveled 


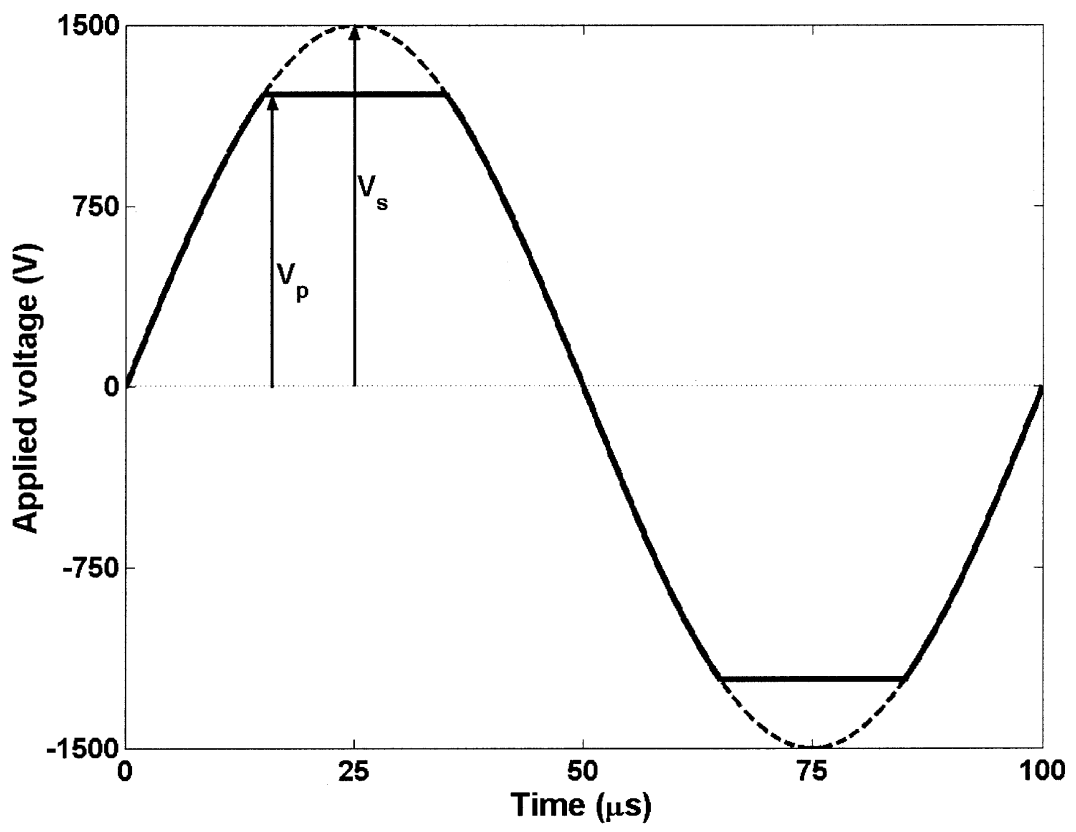

(a)

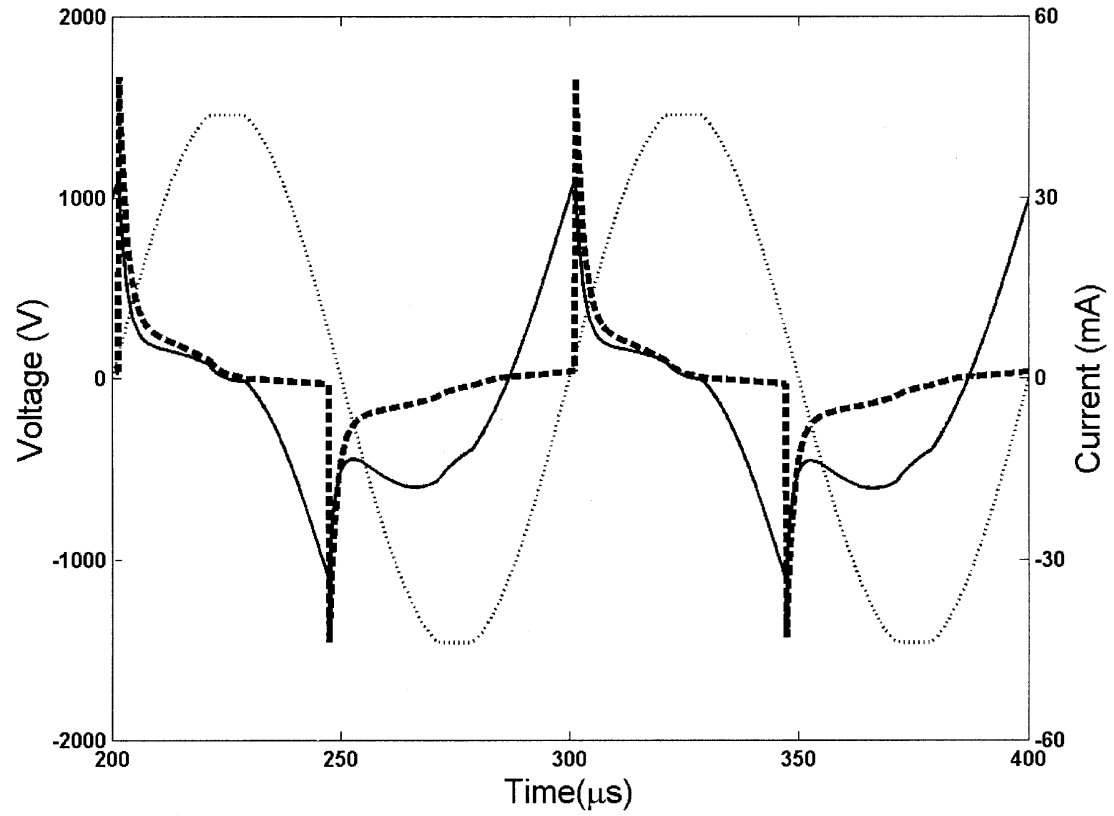

(b)

Fig. 4. (a) Pulsed excitation voltage (solid curve) at 10-kHz repetition frequency, made from a sinusoidal voltage (dashed curve) having its peaks levelled. (b) Time dependence of the peak-levelled excitation voltage (dotted curve), the gas voltage (solid curve), and the discharge current (thick dashed curve),

voltage $V_{p}$ varies from 0.4 to $1.5 \mathrm{kV}$. The repetition frequency of the excitation voltage remains at $10 \mathrm{kHz}$.

Fig. 4(b) shows the voltage and current characteristics of a diffuse nonthermal atmospheric gas discharge generated with $V_{p}=1.2 \mathrm{kV}$. The discharge current remains repetitive and stable through many tens of cycles of the applied voltage. Therefore, under the conditions considered here, pulsing the plasma-generating voltage does not appear to significantly affect the establishment of diffuse nonthermal atmospheric plasma. More specifically, the waveform of the discharge current remains relatively unchanged from that under the sinusoidal excitation, and the peak current is again around $55 \mathrm{~mA}$ very similar to the sinusoidal case of Fig. 2.
The basic $V-I$ characteristics remain relatively unchanged until $V_{p}$ decreases below $750 \mathrm{~V}$ when the generated plasma becomes unsustainable and eventually extinguishes. This is illustrated in Fig. 5, where the normalized electron density and the normalized plasma power density are plotted against the normalized magnitude of the applied voltage, $V_{p} / V_{s}$. It is evident that when $V_{p} / V_{s}>0.5$ plasma pulsing does not significantly affect electron density while the plasma power is reduced by up to $40 \%$. Further numerical simulations suggest that ion densities are also largely unaffected as long as $V_{p} / V_{s}>0.5$. Given that electron and ion densities remain approximately the same and that the discharge current and the gas voltage undergo relatively small changes, the basic plasma 


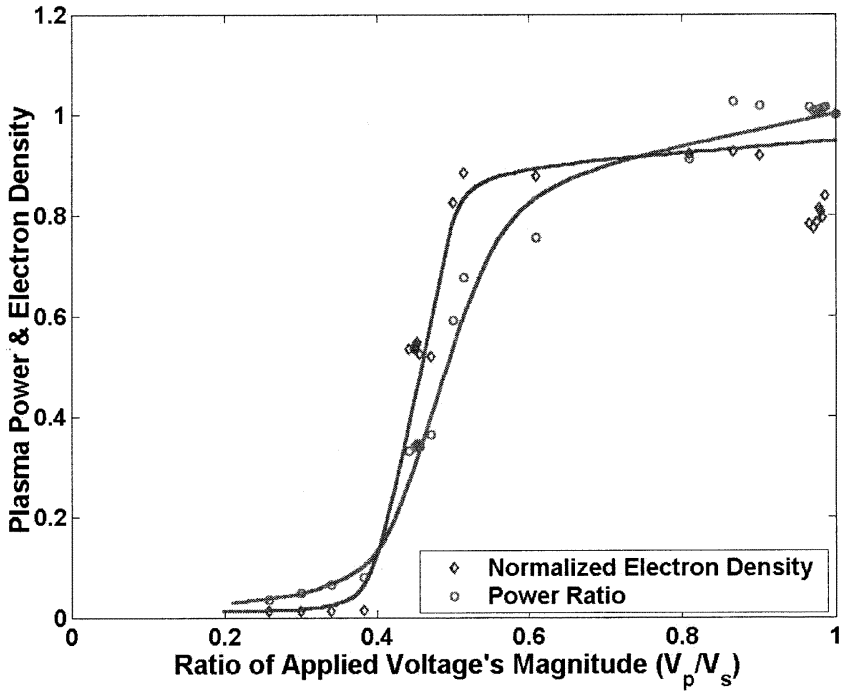

Fig. 5. Normalized plasma power density (circles) and normalized electron density (diamonds) as a function of reduced magnitude of the applied voltage with $0.5 \%$ nitrogen.

characteristics should remain relatively unchanged. In other words, voltage pulsing in Fig. 4 is unlikely to affect adversely plasma-activated applications that rely on the production of electrons or/and ions, yet capable of considerable power saving.

It is found that the amount of power saving is dependent on that of nitrogen impuries. In the case of very little nitrogen impurities $(<0.01 \%)$, numerical studies suggest a power saving of more than 50\%, as indicated in Fig. 6. Again, such significant power saving is achieved without affecting the production of electrons and ions. It is also found that the power saving is not at the expense of the production of excited neutral species either. With $0.5 \%$ nitrogen, Fig. 7 shows the normalized density of $\mathrm{He}\left(2^{3} \mathrm{~S}\right)$ as a function of the $V_{p} / V_{s}$ ratio. It is evident that the trend of the metastable density effectively tracks that of the electron density. So if the plasma power is reduced with the electron density kept approximately the same as that with sinusoidal excitation (without pulsing), the metastable density will also remain at approximately the same level as its value with sinusoidal excitation.

The density tracking between electrons and metastables suggests a key role played by electrons in influencing production of excited neutral species in diffuse atmospheric gas discharges. It is known that energy required to excite neutral species and create chemically reactive species is usually provided through the kinetic energy of electrons. There are also reactive species whose densities are directly proportional to electron density. For example, densities of most oxygen species (e.g., atomic oxygen, singlet-sigma metastable oxygen, singlet-delta metastable oxygen) in a diffuse nonthermal atmospheric discharge in an oxygen-helium mixture are found to proportional to $P_{\mathrm{RF}}^{m}$ where $m=0.8-1.9$ and $P_{\mathrm{RF}}$ is the input RF power to the generated plasma [35]. According to (3), these oxygen densities are also proportional to electron density. Therefore, the similar electron densities in the sinusoidal case of Fig. 2 and the pulsed case of Fig. 4 may be a good basis to consider that the densities of most reactive species in the two cases would be similar and so would their chemical reactivity.

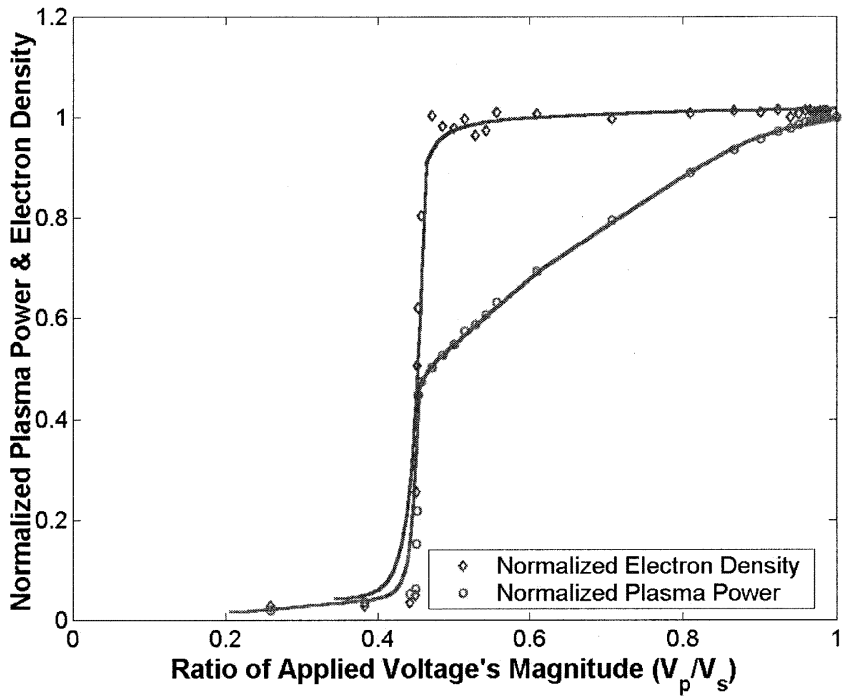

Fig. 6. Normalized plasma power density (circles) and normalized electron density (diamonds) as a function of reduced magnitude of the applied voltage with no nitrogen impurities.

Thus, it is possible to produce at least some reactive species electrically efficiently with voltage pulsing, and as such this will also be useful for applications that reply on reactive species. It is important to note, however, that in general there is no direct correlation between electron density and densities of all reactive species. There are reactive species whose densities may be inversely proportional to electron density, for example ozone in the oxygen-helium plasma mentioned previously [35]. So, for individual diffuse nonthermal atmospheric plasmas, the suggested maintenance of production of reactive species deduced from that of electron production needs to be assessed carefully. Our observation is that it is possible for diffuse nonthermal atmospheric plasmas to maintain densities of some reactive species with reduced electric power. As an additional indication of the similarity between the sinusoidal case and the pulsed case of Fig. 4, temporal and spatial variation of electron density is plotted in Fig. 8. This is similar to that in Fig. 3.

The "tail-off" phase of the applied voltage in Fig. 9(a) (between 25-50 $\mu \mathrm{s}$ ) may be trimmed to further enhance energy efficiency for plasma generation. One way to trim the applied voltage is shown in Fig. 9(a); the $V-I$ characteristics of its induced atmospheric plasma are shown in Fig. 9(b). It is evident that moderate voltage trimming does not significantly affect the generation and characteristics of induced nonthermal atmospheric plasmas, though the discharge current has different peak values in different half cycles. Numerical studies suggest that further power saving is possible but very modest, typically a few percent and at best $10 \%$ before electron density starts to decrease.

We have so far established that generation of diffuse nonthermal atmospheric plasmas can be made more efficient by altering the waveform of the applied voltage without affecting the basic plasma characteristics. Now, we consider how pulsewidth may affect plasma generation. As an example, we consider one particular type of pulsed voltage signals constructed from a sinusoidal signal for the voltage rise phase and a Gaussian decay 


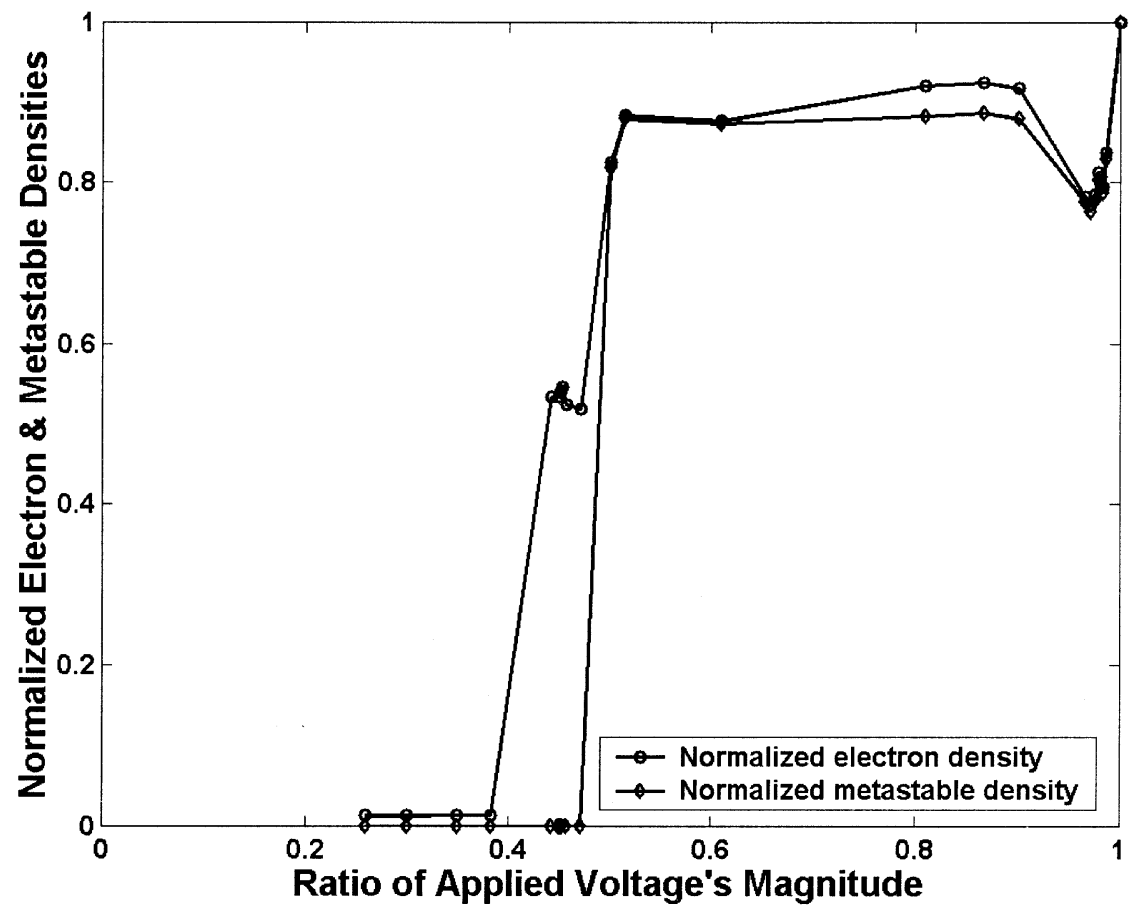

Fig. 7. Normalized electron density (circles) and normalized metastable density (diamonds) as a function of reduced magnitude of the applied voltage with $0.5 \%$ nitrogen impurities.

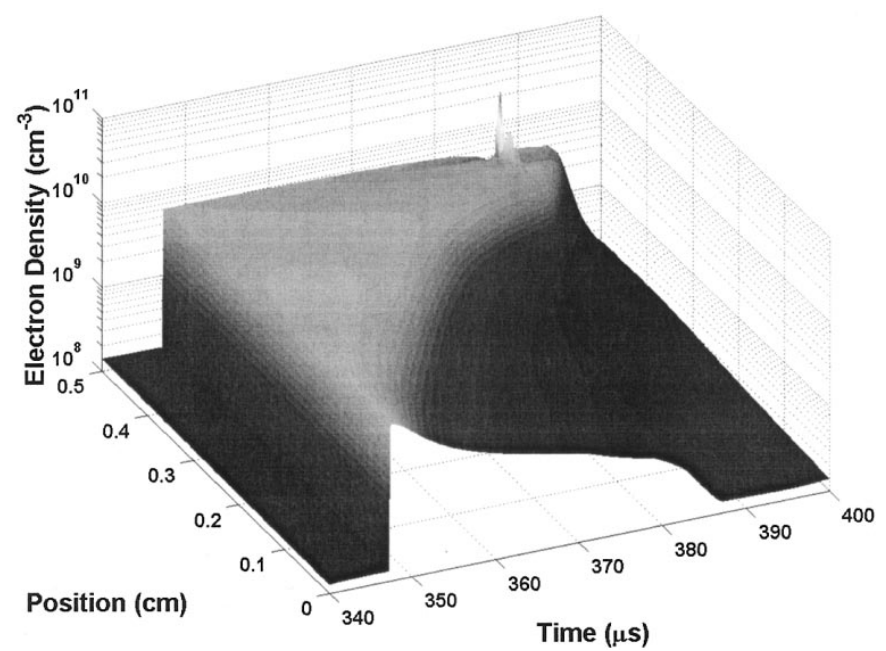

Fig. 8. Variation of electron density between the two electrodes over one-half cycle of the applied voltage of a peak-levelled sinusoidal. The instantaneous anode and cathode are at $x=0$ and $x=0.5 \mathrm{~cm}$, respectively, from $t=350$ to $t=400 \mu \mathrm{s}$.

for the voltage tail phase. Mathematically, in each cycle, they may be expressed in the following:

$$
V(t)=\left\{\begin{array}{l}
V_{0} \sin \omega t, \quad \text { if } 2 N \pi \leq t<t_{0}+2 N \pi \\
V_{p} \exp \left[-\left(t-t_{0}-2 N \pi\right)^{2} / \tau^{2}\right], \\
\quad \text { if } t_{0}+2 N \pi \leq t<2 N \pi+\pi \\
V_{0} \sin \omega t, \quad \text { if } 2 N \pi+\pi \leq t<t_{0}+2 N \pi+\pi \\
-V_{p} \exp \left[-\left(t-t_{0}-2 N \pi-\pi\right)^{2} / \tau^{2}\right], \\
\quad \text { if } t_{0}+2 N \pi+\pi \leq t<2 N \pi+2 \pi
\end{array}\right.
$$

where $V_{0}$ is the peak voltage of the sinusoidal signal, $V_{p}$ is the peak voltage of the Gaussian decay signal, $\tau$ is the pulsewidth of the Gaussian signal, $\omega=2 \pi / T$ is the angular frequency of the applied voltage with $T$ being its period, and $t_{0}$ the instant at which the sinusoidal and the Gaussian signals joint. $T_{0}$ and $t_{0}$ are defined as follows:

$$
\begin{aligned}
T_{0} & =\frac{T}{2 \pi} \arcsin \left[\frac{V_{p}}{V_{0}}\right] \\
t_{0} & =T_{0}+k T / 2 \\
k & =\bmod (t, T / 2) .
\end{aligned}
$$

To compare to the sinusoidal case of Fig. 2, we set $V_{0}=1.5 \mathrm{kV}$, $V_{p}=1.4 \mathrm{kV}$, and $\tau=T / 8$. The waveform of the applied voltage is shown in Fig. 10(a), and the voltage and current signals of the generated atmospheric plasma is shown in Fig. 10(b). It is evident that the pattern of one discharge every half cycle remains and this is repetitive for many tens of cycles. On the other hand, the gas voltage is markedly different from that in the sinusoidal case of Fig. 2. Particularly, it has a large hump preceding the main peak that causes the discharge event in each half cycle. Also, after the discharge event where the discharge current peaks, the gas discharge does not reduce to zero as rapidly as in the sinusoidal case.

The discharge current in Fig. 10(b) is between 30-45 mA, markedly lower than $55 \mathrm{~mA}$ calculated for the sinusoidal case. Interestingly, the pulsewidth of the discharge current is much larger than that in the sinusoidal case (Fig. 2), particularly for the positive half cycles. For the case in Fig. 10, electric power density consumed in the plasma is about $0.32 \mathrm{~W} / \mathrm{cm}^{3}$, about $7 \%$ above that in the sinusoidal case. Further numerical examples studied suggest that under other pulsing conditions diffuse atmospheric helium plasmas excited with the pulsed voltage of Fig. 10(a) consume approximately the same amount of electric power as those with sinusoidal excitation. Therefore, shortening the pulsewidth is not able to achieve energy 


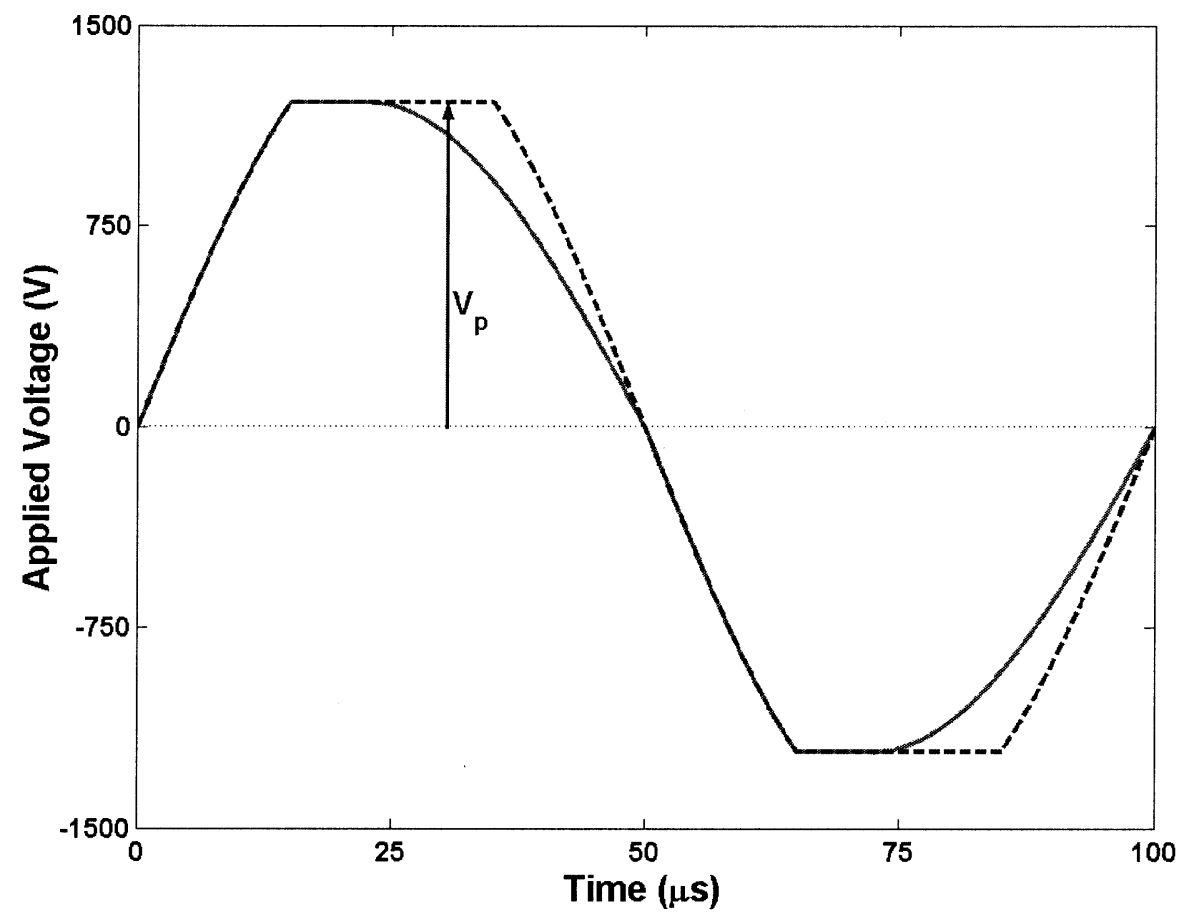

(a)

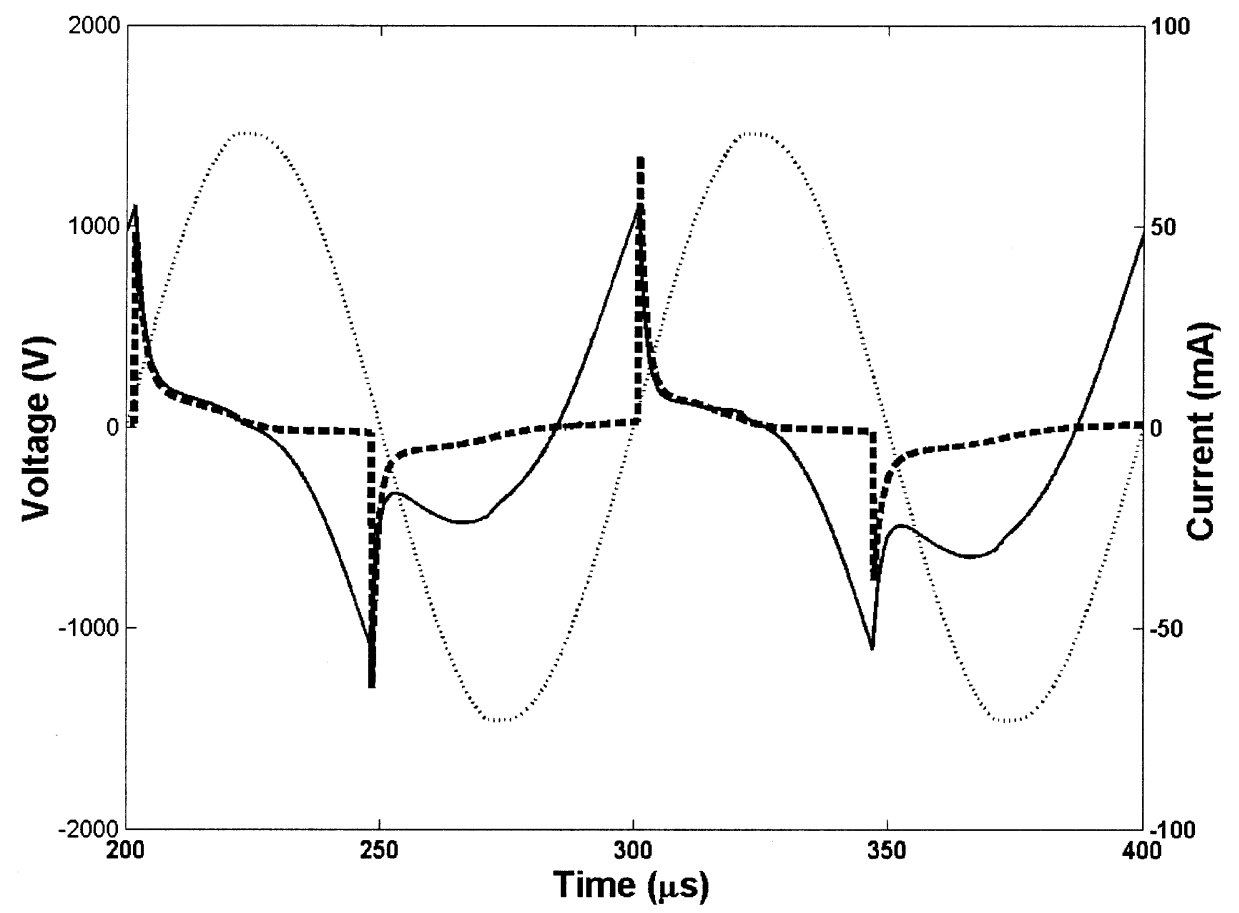

(b)

Fig. 9. (a) Peak-leveled excitation voltage at 10-kHz repetition frequency (dashed curve) and with its pulse tail trimmed (solid curve). (b) Time dependence of the peak-leveled and tail-trimmed excitation voltage (dot curve), the gas voltage (solid curve), and the discharge current (thick dashed curve).

saving. From the $V-I$ characteristic, this is likely due to the wider pulsewidths for both the discharge current and the gas voltage. On the other hand, electron density achievable can be much higher. For the case shown in Fig. 10(b), the peak electron density is found to be $1.74 \times 10^{10} \mathrm{~cm}^{-3}, 83 \%$ above $0.95 \times 10^{10} \mathrm{~cm}^{-3}$ for the sinusoidal case (Fig. 3). Fig. 11 shows spatial variation of electron density over one-half cycle. This shows a similar distribution to that in Figs. 3 and 8, yet with clear difference near the voltage polarity reversal point. There is a clear evidence of pulsed production of electrons. Further numerical calculations suggest that by changing the pulsewidth of the applied voltage in Fig. 10(a), electron and metastable densities can be made much greater than that under sinusoidal excitation. Though inappropriate wave-shaping of the applied voltage can also reduce electron and metastable densities, the previous discussions suggest that through control 


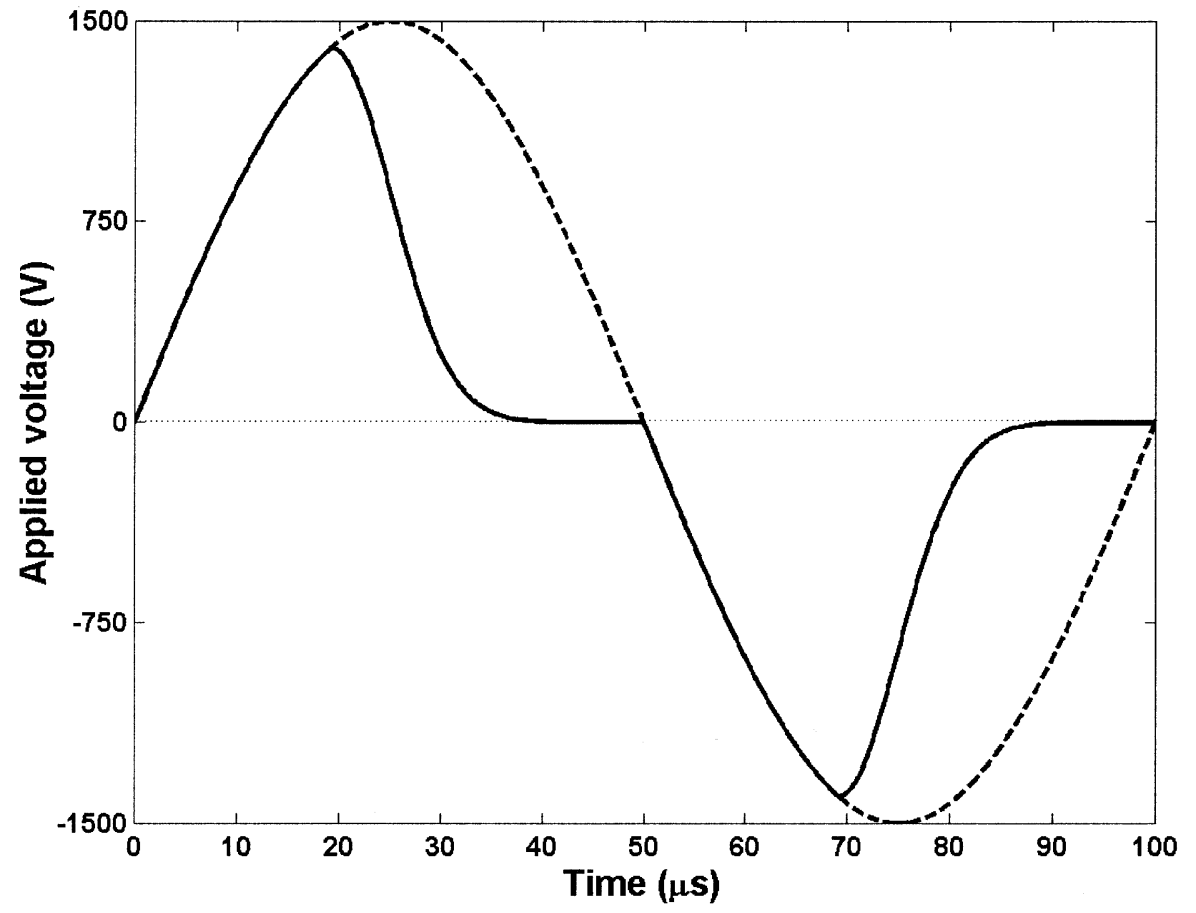

(a)

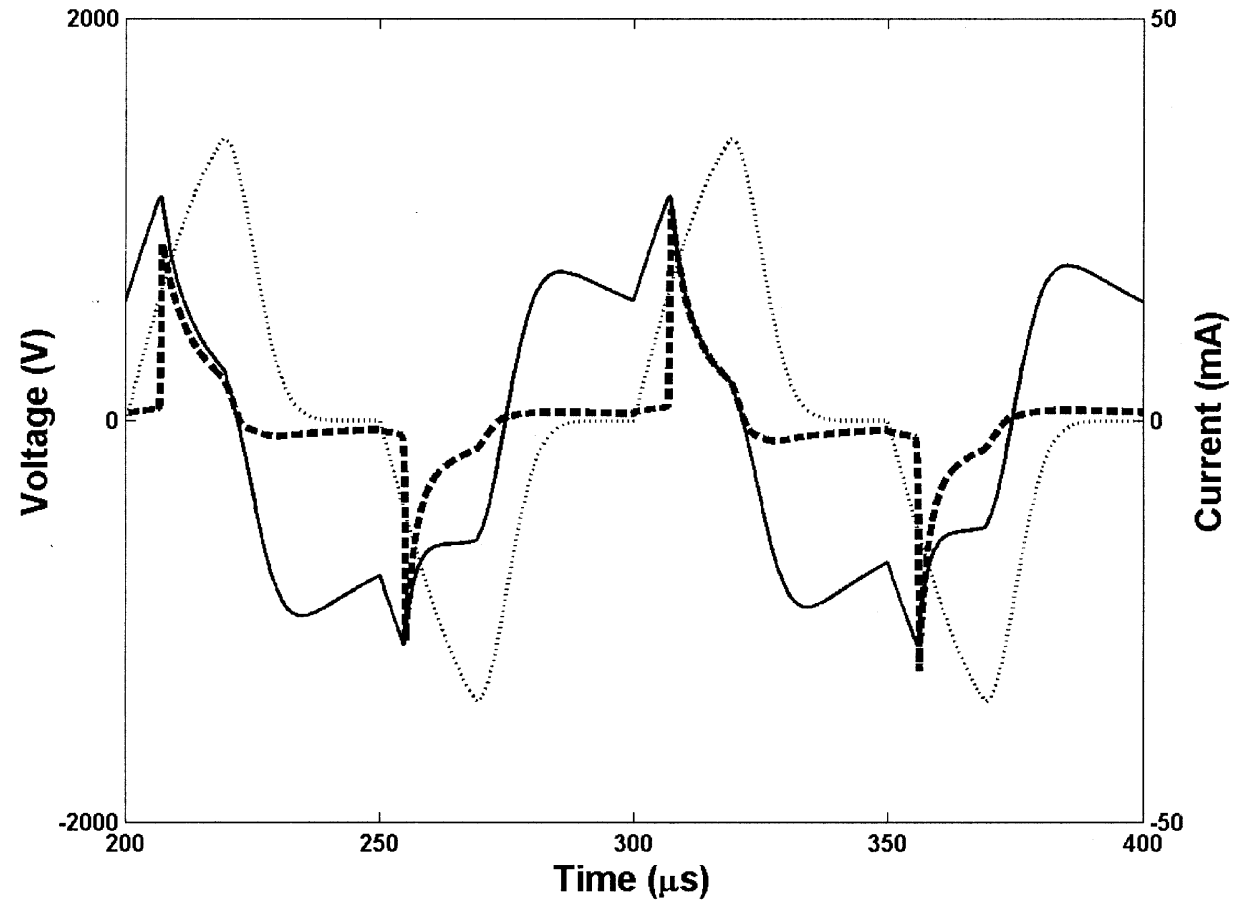

(b)

Fig. 10. (a) Sinusoidal excitation voltage at 10-kHz repetition frequency (dashed curve) and with its tail shaped with a Gaussian decay (solid curve). (b) Time dependence of the excitation voltage (dot curve), the gas voltage (solid curve), and the discharge current (thick dashed curve).

of pulsewidth it is possible to enhance production of electrons and metastables without increasing the plasma power consumption. To see this more clearly, we calculate the ratio of electron density to plasma power density (number of electrons that can be produced at a given input power). This is $5.4 \times 10^{10} / \mathrm{W}$ for the case in Fig. 10 and $3.2 \times 10^{10} / \mathrm{W}$ for the sinusoidal case of Fig. 3, an improvement of $68 \%$. From this comparison, it is still electrically efficient to pulse the applied voltage for the generation of diffuse nonthermal atmospheric plasmas.

\section{CONCLUSION}

Pulsed plasma generation was studied for diffuse nonthermal atmospheric helium discharges using a 1-D computer code. It was found that the electric power needed to sustain nonthermal atmospheric plasmas could be significantly reduced by pulsing the plasma generating voltage. Significantly, this is achieved without affecting the densities of electrons, ions, and metastables. Plasma power can be reduced by more than $50 \%$ by 


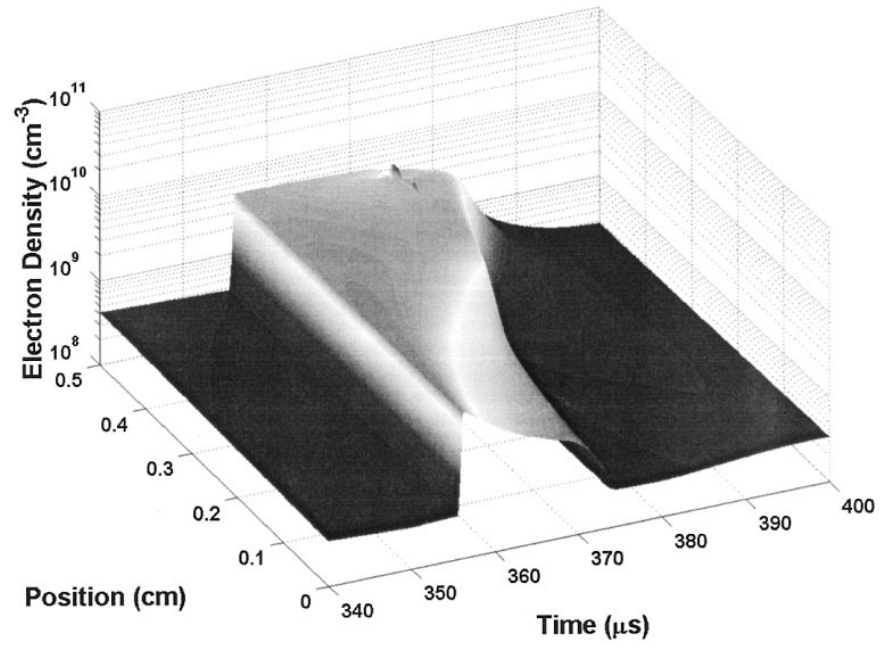

Fig. 11. Variation of electron density between the two electrodes over one-half cycle of a pulsed applied voltage with a Gaussian-shaped tail. The instantaneous anode and cathode are at $x=0$ and $x=0.5 \mathrm{~cm}$, respectively, from $t=350$ to $t=400 \mu \mathrm{s}$.

choosing appropriate pulse shape and pulsewidth, though pulse shape appears to be much more effective. In the case of altering pulsewidth, it was shown that although little power saving can be made the production of electrons and metastables can be improved by $68 \%$. Therefore, effects of waveform-shaping and pulsewidth reducing are different, and so can be used to cater different applications. In general, waveform-shaping should be useful for applications in which power consumption is a key issue, for example industrial-scale materials processing and aircraft cloaking. On the other hand, manipulation of pulsewidth is useful for applications that favor high-density plasmas.

\section{REFERENCES}

[1] B. Eliasson and U. Kogelschatz, "Nonequilibrium volume plasma chemical-processing," IEEE Trans. Plasma Sci., vol. 19, pp. 1063-1077, Dec. 1991.

[2] B. M. Penetrante, J. N. Barsley, and M. C. Hsaio, "Kinetic analysis of nonthermal plasmas used for pollution control," J. Appl. Phys., vol. 36, pp. 5007-5017, 1997.

[3] J. Friedrich, L. Wigant, W. Unger, A. Lippitz, and H. Wittrich, "Corona, spark and combined UV and ozone modification of polymer films WeBP23," Surf. Coat. Technol., vol. 98, pp. 879-885, 1998.

[4] A. C. Gentile and M. J. Kushner, "Reaction chemistry and optimization of plasma remediation of $\mathrm{N}_{x} \mathrm{O}_{y}$ from gas streams," J. Appl. Phys., vol. 78, pp. 2074-2085, 1995.

[5] A. von Engle, R. Seeliger, and M. Steenback, "On the glow discharge at high pressure," Zeit. fur Phys., vol. 85, pp. 144-160, 1933.

[6] M. Baeva, A. Dogan, J. Ehlbeck, A. Pott, and J. Uhlenbusch, "CARS diagnostic and modeling of a dielectric barrier discharge," Plasma Chem. Plasma Process., vol. 19, pp. 445-466, 1999.

[7] R. Hackam and H. Akiyama, "Air pollution control by electrical discharges," IEEE Trans. Dielect. Elect. Insulation, vol. 7, pp. 654-683, Oct. 2000.

[8] E. E. Kunhardt, "Generation of large-volume, atmospheric-pressure, nonequilibrium plasmas," IEEE Trans. Plasma Sci., vol. 28, pp. 189-200, Feb. 2000.

[9] M. J. Shenton and G. C. Stevens, "Surface modification of polymer surfaces: Atmospheric plasma versus vacuum plasma treatments," J. Phys. D, Appl. Phys., vol. 34, pp. 2761-2768, 2001.

[10] F. Massines, A. Rabehi, P. Decomps, R. B. Gadri, P. Segur, and C. Mayoux, "Experimental and theoretical study of a glow discharge at atmospheric pressure controlled by dielectric barrier," J. Appl. Phys., vol. 83, pp. 2950-2957, 1998.
[11] T. C. Montie, K. Kelly-Wintenberg, and J. R. Roth, "An overview of research using a one atmospheric uniform glow discharge plasma (OAUGDP) for sterilization of surfaces and materials," IEEE Trans. Plasma Sci., vol. 28, pp. 41-50, Feb. 2000.

[12] K. Maruyama, I. Tsumagari, M. Kanezawa, Y. Gunji, M. Morita, M. Kogoma, and S. Okazaki, "Preparation of $\mathrm{ZnO}$ films from $\mathrm{Zn}_{2}^{+}$aqueous mist using atmospheric pressure glow plasma," J. Mater. Sci. Lett., vol. 20, pp. 481-484, 2001.

[13] A. Schutze A, J. Y. Jeong, S. E. Babayan, J. Park, and G. S. Selwyn, "The atmospheric-pressure plasma jet: A review and comparison to other plasma sources," IEEE Trans Plasma Sci, vol. 26, pp. 1685-1694, Dec. 1998.

[14] J. Park, I. Henins, W. Hermann, and G. S. Selwyn, "Gas breakdown in an atmospheric pressure radio-frequency capacitive plasma source," $J$. Appl. Phys., vol. 89, pp. 15-19, 2001.

[15] X. T. Deng and M. G. Kong, "Parametric conditions for generation of stable atmospheric pressure nonthermal plasmas," in Proc. 2001 Ann. Rep., Conf. Electrical Insulation and Dielectric Phenomena 677, IEEE Catalogue Number: 01CH37225, 2001, pp. 677-680.

[16] F. Tochikubo, T. Chiba, and T. Watanabe, "Structure of low-frequency helium glow discharge at atmospheric pressure between parallel plate dielectric electrodes," Jpn J. Appl. Phys., vol. 38, pp. 5244-5250, 1999.

[17] S. Patankar, Numerical Heat Transfer and Fluid Flow. Bristol, PA: Hemisphere, 1980.

[18] A. L. Ward, "Calculations of cathode-fall characteristics," J. Appl. Phys., vol. 33, pp. 2789-2794, 1964.

[19] J. Dutton, "A survey of electron swarm data," J. Phys. Chem. Ref. Data, vol. 4, pp. 577-856, 1975.

[20] I. Peres, N. Ouadoudi, L. C. Pitchford, and J. P. Boeuf, "Analytical formulation of ionization source term for discharge models in argon, helium, nitrogen, and silane," J. Appl. Phys., vol. 72, pp. 4533-4536, 1992.

[21] R. Ben Gadri, "Numerical simulation of an atmospheric pressure and dielectric barrier controlled glow discharge," Ph.D. dissertation, Univ. Paul Sabatier, Toulouse, France, 1997.

[22] J. M. Pouvesle, A. Bouchoule, and J. Stevefelt, "Modeling of the charge transfer afterglow excited by intense electrical discharges in high pressure helium nitrogen mixtures," J. Chem. Phys., vol. 77, pp. 817-820, 1982.

[23] R. Deloche, P. Monchicourt, M. Cheret, and F. Lambert, "High-pressure helium afterglow at room temperature," Phys. Rev. A, Gen. Phys., vol. 13, pp. 1140-1176, 1976.

[24] A. Cenian, A. Chernukho, and V. Borodin, "Modeling of plasma-chemical reactions in gas mixture of $\mathrm{CO}_{2}$ laser," Contrib. Plasma Phys., vol. 35, pp. 273-296, 1995.

[25] L. L. Alves, G. Gousset, and C. M. Ferreira, "A collisional-radiative model for microwave discharges in helium at low and intermediate pressures," J. Phys. D, Appl. Phys., vol. 25, pp. 1713-1732, 1992.

[26] T. Quinteros, H. Gao, D. R. DeWitt, R. Schuch, S. Pajek, S. Asp, and D. Belkic, "Recombination of $D^{+}$and $\mathrm{He}^{+}$ions with low energy free electrons," Phys. Rev. A, Gen. Phys., vol. 51, pp. 1340-1346, 1995.

[27] P. C. Hill and P. R. Herman, "Reaction processes in a $\mathrm{He}_{2}^{+}\left({ }^{2} \Pi_{u} \rightarrow\right.$ $a_{2} \Sigma_{g}^{+}$) flash lamp," Phys. Rev. A, Gen. Phys., vol. 47, pp. 4837-4844, 1993 .

[28] I. A. Kossyl, A. Yu Kostinsky, A. A. Matveyev, and V. P. Silakov, "Kinetic scheme of the nonequilibrium discharge in nitrogen-oxygen mixtures," Plasma Source Sci. Technol., vol. 1, pp. 207-220, 1992.

[29] A. von Engel, Ionized Gases. New York: AIP, 1994, ch. 3.

[30] J. R. Roth, Industrial Plasma Engineering. Bristol, U.K.: IOP, 1995, vol. 1 , ch. 12 .

[31] T. Yokoyama, M. Kogoma, T. Moriwaki, and S. Okazaki, "The mechanism of the sterilization of glow plasmas at atmospheric pressure," $J$. Phys. D, Appl. Phys., vol. 23, pp. 1125-1128, 1990.

[32] L. Mangolini, K. Orlov, U. Kortshagen, H. Heberlein, and U. Kogelschatz, "Radial structure of low-frequency atmospheric-pressure glow discharge in helium," Appl. Phys. Lett., vol. 80, pp. 1722-1724, 2002.

[33] Z. Chen, J. E. Morrison, R. Ben Gadri, and J. R. Roth, "A low-frequency impedance matching circuit for a one atmospheric uniform glow discharge plasma reactor," presented at the 25th IEEE Int. Conf. Plasma Science, Raleigh, NC, June 1998. Pap. no. 6P63.

[34] R. H. Stark and K. H. Schoenbach, "Direct current high-pressure glow discharges," J. Appl. Phys., vol. 85, pp. 2075-2080, 1999.

[35] J. Y. Jeong, J. Park, I. Henins, S. E. Babayan, V. J. Tu, G. S. Selwyn, G. Ding, and R. F. Hicks, "Reaction chemistry in the afterglow of an oxygen-helium atmospheric plasma," J. Phys. Chem., vol. 104, pp. 8027-8032, 2000. 


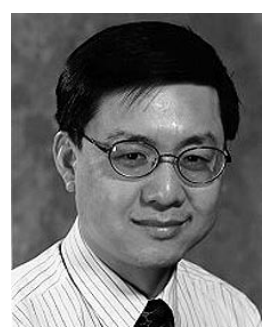

Michael G. Kong (M'94, SM'98) received the B.Sc. and M.Sc. degrees in electronics engineering from Zhejiang University, Hangzhou City, China, in 1984 and 1987, respectively, and the Ph.D. degree in electrical engineering from Liverpool University, Liverpool, U.K., in 1992.

From 1995 and 1999, he was a Lecturer at Liverpool University. He has been a Senior Lecturer at Loughborough University, Leicestershire, U.K., since 1999. He is a Committee Member of the Ion and Plasma Surface Interaction Group, Institute of Physics, U.K., and a Chief Examiner for the Engineering Council, U.K. He has been actively involved in research on nonthermal atmospheric gas discharges, compact waveguide free-electron lasers, and power systems and devices. In these subject areas, he has authored some 100 papers published in scientific journals and peer-reviewed conference proceedings.

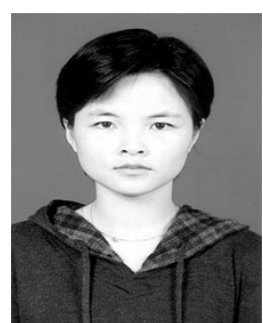

Xu Tao Deng (S'02) received the B.Eng. degree in electronic engineering from Shenzhen University, Shenzhen City, China, in 1999, and the M.Sc. (Eng.) degree in intelligence engineering from the University of Liverpool, Liverpool, U.K., in 2000. She is currently working toward the Ph.D. degree at Loughborough University, Leicestershire, U.K.

Her present interest is the development of PC-based computer codes for nonthermal atmospheric pressure discharges. 